\title{
Corrigendum: Whole Genome Sequencing of Australian Candida glabrata Isolates Reveals Genetic Diversity and Novel Sequence Types
}

\begin{abstract}
Chayanika Biswas ${ }^{1,2}$, Vanessa R. Marcelino ${ }^{2,3,4 t}$, Sebastiaan Van Hal ${ }^{5 t}$, Catriona Halliday ${ }^{6}$, Elena Martinez ${ }^{1,2}$, Qinning Wang ${ }^{1,2}$, Sarah Kidd ${ }^{7}$, Karina Kennedy ${ }^{8}$, Deborah Marriott ${ }^{9}$, C. Orla Morrissey ${ }^{10}$, Ian Arthur ${ }^{11}$, Kerry Weeks ${ }^{12}$, Monica A. Slavin ${ }^{13}$, Tania C. Sorrell ${ }^{2,3,4}$, Vitali Sintchenko ${ }^{1,2,3,4 \neq}$, Wieland Meyer ${ }^{1,2,3,4 \neq}$ and Sharon C.-A. Chen ${ }^{1,2,3,4,6 * \neq}$

${ }^{1}$ Centre for Infectious Diseases and Microbiology-Public Health, Westmead Hospital, Sydney, NSW, Australia, ${ }^{2}$ Westmead Clinical School, Faculty of Medicine and Health, The University of Sydney, Sydney, NSW, Australia, ${ }^{3}$ Centre for Infectious Diseases and Microbiology, Westmead Institute for Medical Research, Westmead, NSW, Australia, ${ }^{4}$ Marie Bashir Institute for Emerging Infectious Diseases and Biosecurity, The University of Sydney, Sydney, NSW, Australia, ${ }^{5}$ Department of Infectious Diseases and Microbiology, New South Wales Health Pathology, Royal Prince Alfred Hospital, Faculty of Medicine and Health, The University of Sydney, Sydney, NSW, Australia, ${ }^{6}$ Centre for Infectious Diseases and Microbiology Laboratory Services, ICPMR, New South Wales Health Pathology, Westmead Hospital, Sydney, NSW, Australia, ${ }^{7}$ National Mycology Reference Centre, SA Pathology, Adelaide, SA, Australia, ${ }^{8}$ Department of Microbiology and Infectious Diseases, Canberra Hospital \& Health Services, Australian National University Medical School, Canberra, ACT, Australia, ${ }^{9}$ Department of Microbiology and Infectious Diseases, St Vincent's Hospital, Sydney, NSW, Australia, ${ }^{10}$ Department of Infectious Diseases, Alfred Health and Monash University, Melbourne, VIC, Australia, ${ }^{11}$ Department of Microbiology, PathWest Laboratory Medicine, Queen Elizabeth II Medical Centre, Perth, WA, Australia, ${ }^{12}$ Department of Microbiology and Infectious Diseases, Royal North Shore Hospital, Sydney, NSW, Australia, ${ }^{13}$ National Centre for Infections in Cancer, Peter MacCallum Cancer Centre, Melbourne, VIC, Australia
\end{abstract}

Keywords: whole genome sequencing, Candida glabrata, MLST, sequence type, Australia

\section{A Corrigendum on}

Whole Genome Sequencing of Australian Candida glabrata Isolates Reveals Genetic Diversity and Novel Sequence Types

by Biswas, C., Marcelino, V. R., Van Hal, S., Halliday, C., Martinez, E., Wang, Q., et al. (2018). Front. Microbiol. 9:2946. doi: 10.3389/fmicb.2018.02946

In the original article, there were a small number of transcription errors in Figure $\mathbf{1}$ as published.

There are:

The label "Norway 6" (at position 6 o'clock) was incorrectly "positioned" with isolate WM_18.31 (ST45) and should have been positioned with "Norway 5." This is now corrected.

Isolates Taiwan 1 and Taiwan 2 should be positioned together. In addition, the isolate WM_18.57 was inadvertently omitted from the ST16 "bubble."

Isolate WM_18.51 should have been placed in the group under ST46 instead of ST59. The corrected Figure 1 appears below.

The authors apologize for these errors and state that they do not change the scientific findings of the article in any way. The original article has been updated.

Copyright (๐) 2019 Biswas, Marcelino, Van Hal, Halliday, Martinez, Wang, Kidd, Kennedy, Marriott, Morrissey, Arthur, Weeks, Slavin, Sorrell, Sintchenko, Meyer and Chen. This is an open-access article distributed under the terms of the Creative Commons Attribution License (CC BY). The use, distribution or reproduction in other forums is permitted, provided the original author(s) and the copyright owner(s) are credited and that the original publication in this journal is cited, in accordance with accepted academic practice. No use, distribution or reproduction is permitted which does not comply with these terms. 


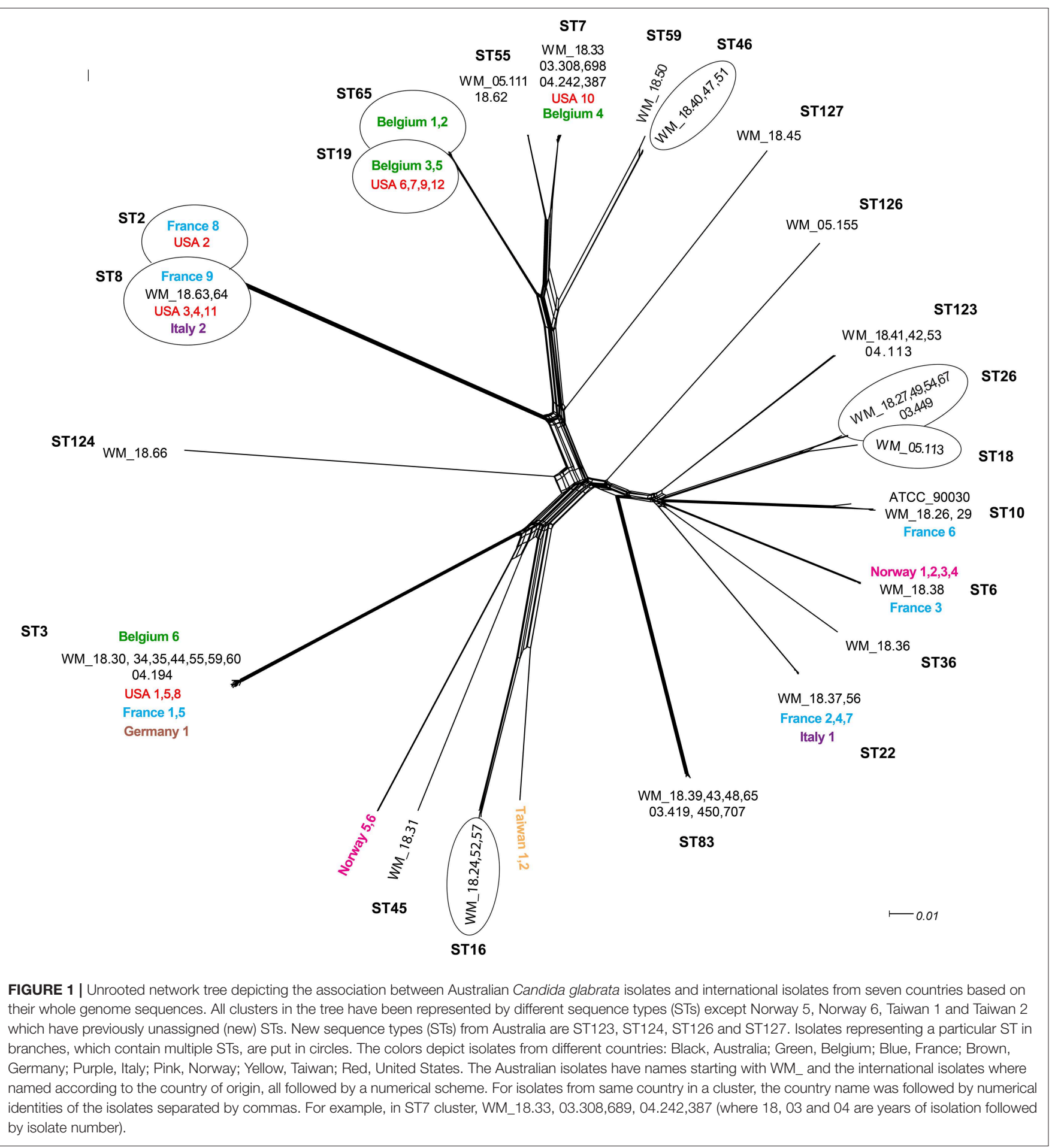

